\title{
Development and characterization of nuclear microsatellite markers to reveal the neutral demographic background of flower color polymorphism in Geranium thunbergii (Geraniaceae)
}

\author{
Seikan Kurata ${ }^{1 *}$, Shota Sakaguchi ${ }^{2}$, Hitomi Mishima ${ }^{1}$, \\ Takashi Tsuchimatsu ${ }^{3}$ and Motomi Ito ${ }^{1}$ \\ ${ }^{1}$ Department of General Systems Studies, Graduate School of Arts and Sciences, \\ University of Tokyo, Meguro, Tokyo 153-8902, Japan \\ ${ }^{2}$ Department Graduate School of Human and Environmental Studies, \\ Kyoto University, Kyoto, Kyoto 606-8501, Japan \\ ${ }^{3}$ Department of Biological Sciences, Graduate School of Science, \\ University of Tokyo, Bunkyo, Tokyo 113-0033, Japan
}

(Received 11 November 2020, accepted 15 January 2021; J-STAGE Advance published date: 22 April 2021)

Nuclear microsatellite markers were developed for Geranium thunbergii, an herbaceous plant characterized by petal color polymorphism. Utilizing RNA sequencing data obtained by next-generation sequencing techniques, we developed and characterized 19 polymorphic microsatellite markers with two to 12 alleles in the nuclear genome. These markers will be used to reveal the genetic structure and demographic history of $G$. thunbergii in the Japanese archipelago, which will elucidate the genetic background of flower color polymorphism among populations.

Key words: flower color polymorphism, genetic diversity, Geranium thunbergii, microsatellite marker, population genetics

Plants are often characterized by petal color variation within and among populations; this phenomenon is defined as flower color polymorphism. Flower color polymorphism has long been of interest to ecologists and evolutionists (Darwin, 1862; Forsman, 2016). For species with intraspecific floral color variation [e.g., Antirrhinum majus L. (Plantaginaceae), Aquilegia coerulea E. James (Ranunculaceae), Dactylorhiza sambucina (L.) Soó (Orchidaceae), Lysimachia arvensis (L.) U. Manns \& Anderb. (Primulaceae) and Raphanus sativus L. (Brassicaceae)], the mechanisms underlying the maintenance of floral diversity have been rigorously investigated (Gigord et al., 2001; Jones and Reithel, 2001; Arista et al., 2013; McCall et al., 2013; Thairu and Brunet,

Edited by Yoshihiko Tsumura

* Corresponding author. seikan.kurata@gmail.com DOI: https://doi.org/10.1266/ggs.20-00062

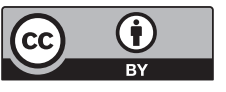

Copyright: @2021 The Author(s). This is an open access article distributed under the terms of the Creative Commons BY 4.0 International (Attribution) License (https://creativecommons.org/ licenses/by/4.0/legalcode), which permits the unrestricted distribution, reproduction and use of the article provided the original source and authors are credited.
2015). Based on these investigations, biotic and abiotic elements (e.g., negative frequency-dependent selection, pollinator preference and temperature) are suggested to be important determinants of flower color polymorphism (Narbona et al., 2018). Selectively neutral factors, i.e., genetic drift and gene flow, have also been implicated in the maintenance of flower color polymorphism (Wright, 1978; Narbona et al., 2018). Therefore, it is important to evaluate the relative contributions of different evolutionary forces in maintaining floral color polymorphism within and among populations.

Geranium thunbergii Siebold ex Lindl. \& Paxton (Geraniaceae) is a perennial herb distributed throughout the Japanese archipelago. The habitats of this species are lowland forest edges and grassy areas. The species shows a geographic cline in floral color: individuals having purple petals (i.e., purple flowers) are distributed on the western side of the Japanese archipelago, while those having white or pale pink petals (i.e., considered as "white flowers") are distributed on the eastern side (Akiyama, 2001; Kadota, 2016). Both flower colors (i.e., purple and white) co-occur in central Honshu, the geographically intermediate distribution area of the species. As such, G. thunbergii has floral color variation both within and 
among populations, and is therefore a suitable plant species for exploring the formation and maintenance mechanisms of flower color polymorphism. In particular, since the geographic distribution pattern of floral color may be related to past neutral demographic history, phylogeographic and population genetics approaches are nec- essary to reveal the geographic pattern of flower color polymorphism. Tsuchimatsu et al. (2014) indicated that selective pressure by herbivores on white flowers and the anthocyanin-mediated herbivore defense of purple flowers are associated with the flower color polymorphism of G. thunbergii, but a more detailed study (e.g., including

Table 1. Characteristics of 19 microsatellite markers developed for G. thunbergii

\begin{tabular}{|c|c|c|c|c|c|c|}
\hline Locus & Primer sequence $\left(5^{\prime}-3^{\prime}\right)$ & $\begin{array}{l}\text { Size range } \\
\quad(b p)\end{array}$ & Motif & BLASTX top hit description [species] & $E$-value & ONEKP ID \\
\hline VKGP_202 & $\begin{array}{l}\text { F: CAGCAAGCACCATGTTACCC } \\
\text { R: AGTGAAGCTCAAGAGAAGCG }\end{array}$ & $150-153$ & $(\mathrm{AG})_{9}$ & $\begin{array}{l}\text { UDP-galactose transporter } 2 \text { isoform X1 } \\
\text { [Carex littledalei }]\end{array}$ & $2.00 \mathrm{E}-63$ & VKGP-2000202 \\
\hline VKGP_2694 & $\begin{array}{l}\text { F: } \text { TCTCCTCCTTCCATTGCCTG } \\
\text { R: CGACCTTCACACAGCAATCC }\end{array}$ & 318-339 & $(\mathrm{AG})_{10}$ & $\begin{array}{l}\text { kinesin-like protein KIN-4A isoform X1 } \\
\text { [Ricinus communis }]\end{array}$ & 0 & VKGP-2002694 \\
\hline VKGP_3319 & $\begin{array}{l}\text { F: CAGAAACAGAGACCATAGCGTC } \\
\text { R: TGTCTGCGAAGAGAGTACGG }\end{array}$ & $167-169$ & $(\mathrm{AG})_{9}$ & $\begin{array}{c}\text { hypothetical protein EZV62_019291 } \\
\text { [Acer yangbiense] }\end{array}$ & $5.00 \mathrm{E}-109$ & VKGP-2003319 \\
\hline VKGP_4952 & $\begin{array}{l}\text { F: AACCAGACCCTTGTAGCTCC } \\
\text { R: CTTTGGAGCTCATTTGAACGTG }\end{array}$ & $326-330$ & $(\mathrm{AC})_{9}$ & No hit & & VKGP-2004952 \\
\hline VKGP_10356 & $\begin{array}{l}\text { F: } \text { TCCTTTCTTCCTTGGTTTCCTG } \\
\text { R: }\end{array}$ & $183-197$ & $(\mathrm{AG})_{9}$ & protease Do-like 7 [Ziziphus jujuba] & 0 & VKGP-2010356 \\
\hline VKGP_14936 & $\begin{array}{l}\text { F: TAGACCCAATTCAGCCTCGG } \\
\text { R: CTCACCAGTTTCCGATTCGC }\end{array}$ & $269-281$ & $(\mathrm{AAC})_{10}$ & $\begin{array}{l}\text { F-box/LRR-repeat protein } 4 \\
\text { [Syzygium oleosum }]\end{array}$ & $3.00 \mathrm{E}-20$ & VKGP-2014936 \\
\hline VKGP_19219 & $\begin{array}{l}\text { F: ATGCGAAGGTGGAGAAGACG } \\
\text { R: } \text { TCTCTCGGCCTGATACAGTG }\end{array}$ & $257-266$ & $(\mathrm{AG})_{8}$ & $\begin{array}{l}\text { DUF502 domain-containing protein } \\
\text { [Cephalotus follicularis }]\end{array}$ & $5.00 \mathrm{E}-11$ & VKGP-2019219 \\
\hline VKGP_25965 & $\begin{array}{l}\text { F: GTTAAGAATGCGGGCGGTAG } \\
\text { R: AGCAAAGCGAATGTCTCACG }\end{array}$ & $334-337$ & $(\mathrm{ATC})_{8}$ & $\begin{array}{c}\text { hypothetical protein FH972_002063 } \\
\text { [Carpinus fangiana] }\end{array}$ & $8.00 \mathrm{E}-112$ & VKGP-2025965 \\
\hline VKGP_26672"1 & $\begin{array}{l}\text { F: TGTTTCTGTTCCGTTGACCC } \\
\text { R: AAGCTCCCATCTCCGATTCC }\end{array}$ & $102-124$ & $(\mathrm{AG})_{9}$ & $\begin{array}{c}\text { probable glutathione peroxidase } 4 \\
\text { [Rhodamnia argentea }]\end{array}$ & $9.00 \mathrm{E}-95$ & VKGP-2026672 \\
\hline VKGP_29328 & $\begin{array}{l}\text { F: GCATTCCTACACAGCATCGG } \\
\text { R: ATCCCAGAGGTGCAGACAAG }\end{array}$ & $211-214$ & $(\mathrm{ATC})_{17}$ & $\begin{array}{c}\text { hypothetical protein CISIN_1g018444mg } \\
\text { [Citrus sinensis] }\end{array}$ & $4.00 \mathrm{E}-87$ & VKGP-2029328 \\
\hline VKGP_31098 & $\begin{array}{l}\text { F: GCAGATTGGAATGTTGGTGC } \\
\text { R: TTGCAAAGCCATCACCCATG }\end{array}$ & $370-376$ & $(\mathrm{AGC})_{9}$ & No hit & & VKGP-2031098 \\
\hline VKGP_31943 & $\begin{array}{l}\text { F: GCATTACGTACACTGGCTGG } \\
\text { R: GGATCCGACCTCCCAAATCC }\end{array}$ & $263-266$ & $(\mathrm{AAC})_{8}$ & ataxin-2 homolog [Malus domestica] & $7.00 \mathrm{E}-06$ & VKGP-2031943 \\
\hline VKGP_32221 & $\begin{array}{l}\text { F: GAGTGAGCAGAGTCTCGAGG } \\
\text { R: AGACGGAGACAGAGCTTCTC }\end{array}$ & $399-427$ & $(\mathrm{AG})_{9}$ & $\begin{array}{l}\text { two-component response regulator ARR12-like } \\
{[\text { Juglans regia }]}\end{array}$ & 0 & VKGP-2032221 \\
\hline VKGP_87603 $3^{* 1}$ & $\begin{array}{l}\text { F: CCGACAGAGAAGCTACGAAC } \\
\text { R: TCGTGACTCAGTGACCTTCC }\end{array}$ & $123-134$ & $(\mathrm{AG})_{9}$ & No hit & & VKGP-2087603 \\
\hline VKGP_92431 $1^{* 1,2}$ & $\begin{array}{l}\text { F: AAGCAGAGAGGTCGATCGAG } \\
\text { R: AGTGTGTGAGAGACTGTACGG }\end{array}$ & $131-141$ & $(\mathrm{AG})_{9}$ & No hit & & VKGP-2092431 \\
\hline VKGP_108374 ${ }^{* 1}$ & $\begin{array}{l}\text { F: CAGACGCGGACAAAGCTAAG } \\
\text { R: TGAACAGCGGGTAAAGAGAG }\end{array}$ & $168-172$ & $(\mathrm{AG})_{9}$ & No hit & & VKGP-2108374 \\
\hline VKGP_108676 ${ }^{* 1}$ & $\begin{array}{l}\text { F: GAGCAGGAGAGAGAAGCAATC } \\
\text { R: AGCAGTTCGTGTACATTGCG }\end{array}$ & $129-141$ & $(\mathrm{AG})_{9}$ & hypothetical protein [Gossypium harknessii] & $7.00 \mathrm{E}-44$ & VKGP-2108676 \\
\hline YGCX_17221 & $\begin{array}{l}\text { F: AGAGGGACCAAACCACTGTC } \\
\text { R: AGGTCAGTGCATGTAGAGGC }\end{array}$ & $160-181$ & $(\mathrm{AAC})_{9}$ & $\begin{array}{c}\text { hypothetical protein GH714_022102 } \\
\text { [Hevea brasiliensis] }\end{array}$ & $3.00 \mathrm{E}-49$ & YGCK-2017221 \\
\hline YGCX_28878 & $\begin{array}{l}\text { F: ACACTCCTTCCCATGATCCG } \\
\text { R: TCTTCTACGCCAACCACCTC }\end{array}$ & $395-419$ & $(\mathrm{ATC})_{9}$ & $\begin{array}{c}\text { uncharacterized protein LOC109022334 } \\
\text { [Juglans regia] }\end{array}$ & $6.00 \mathrm{E}-09$ & YGCX-2028878 \\
\hline
\end{tabular}

\footnotetext{
${ }^{* 1}$ These primers were previously reported as monomorphic microsatellite primers of G. soboliferum var. kiusianum (Kurata et al., 2017).

${ }^{* 2}$ Primer pair VKGP_92431 amplifies two independent loci, and we confirmed that the two loci are in linkage disequilibrium. However, only one locus was adopted in this study; the other locus often showed a null allele, which could have been derived from PCR amplification error.
} 
migration history) is required to elucidate the geographic pattern of flower color polymorphism in G. thunbergii. In this study, we developed polymorphic microsatellite markers for $G$. thunbergii to reveal its population genetics and phylogeographic history.

Assembled RNA sequencing data of G. carolinianum L. and G. maculatum Dum. Cours. were obtained from the ONEKP: BLAST for 1,000 Plants repository (https:// db.cngb.org/onekp/). A similarity search of the contigs against the National Center for Biotechnology Information non-redundant protein database was conducted using the BLASTX algorithm (Altschul et al., 1990) with an $E$-value cutoff of $1.0 \mathrm{E}-5$. We screened the sequences with microsatellite regions for $\geq 8$ dinucleotide repeats and $\geq 8$ trinucleotide repeats using MSATCOMMANDER (Faircloth, 2008), and designed primers using Primer3 software (Rozen and Skaletsky, 2000). A total of 369 primer pairs bordering microsatellites were designed and 141 pairs were selected for PCR amplification trials, using eight individuals from eight populations (Kodaira, Hokkaido Prefecture (Pref.); Saku, Nagano Pref.; Hachioji, Tokyo Pref.; Katsuura, Chiba Pref.; Taki, Mie Pref.; Okayama, Okayama Pref.; Amakusa, Kumamoto
Pref.; and Yakushima, Kagoshima Pref.). For all loci, the forward primer was synthesized with one of three different tag sequences (5'-CACGACGTTGTAAAACGAC-3', 5'-TGTGGAATTGTGAGCGG-3' or 5'-CTATAGGGCACGCGTGGT-3') and the reverse primer was tagged with a pig-tail sequence (5'-GTTTCTT-3') (Brownstein et al., 1996). Genomic DNA was extracted from dried leaves using a modified CTAB method (Milligan, 1992). PCR amplification was carried out following the standard protocol of the Qiagen Multiplex PCR Kit (Qiagen, Hilden, Germany) in a final reaction volume of $10 \mu$ l containing approximately $5 \mathrm{ng}$ of DNA, $5 \mu \mathrm{l}$ of $2 \times$ Multiplex PCR Master Mix, $0.01 \mu \mathrm{M}$ forward primer, $0.2 \mu \mathrm{M}$ reverse primer, and $0.1 \mu \mathrm{M}$ M13 primer (fluorescently labeled with Beckman Dye; Beckman Coulter, Brea, CA, USA). The PCR thermal profile involved denaturation at $95{ }^{\circ} \mathrm{C}$ for $3 \mathrm{~min}$, followed by 35 cycles of $95{ }^{\circ} \mathrm{C}$ for $30 \mathrm{~s}$, $54{ }^{\circ} \mathrm{C}$ for $3 \mathrm{~min}$, and $68^{\circ} \mathrm{C}$ for $1 \mathrm{~min}$, and a final 20 -min extension step at $68{ }^{\circ} \mathrm{C}$. PCR products were loaded onto an auto sequencer (GenomeLab GeXP; Beckman Coulter) to assess fragment lengths using Fragment Analysis Software ver. 8.0 (Beckman Coulter).

The extracted DNA of 23 individuals from Hachioji

Table 2. Genetic diversity of the 19 polymorphic markers for G. thunbergii

\begin{tabular}{|c|c|c|c|c|c|c|c|c|c|c|c|c|c|c|c|c|}
\hline \multirow[t]{2}{*}{ Locus } & \multicolumn{4}{|c|}{ Hachioji $(n=23)$ [white] } & \multicolumn{4}{|c|}{ Mifune $(n=27)$ [purple] } & \multicolumn{4}{|c|}{$\begin{array}{c}\text { Nantan }(n=30) \\
\text { [purple/white] }\end{array}$} & \multicolumn{4}{|c|}{ Total } \\
\hline & $A$ & $A r$ & $H_{\mathrm{e}}$ & $H_{\mathrm{o}}$ & $A$ & $A r$ & $H_{\mathrm{e}}$ & $H_{\mathrm{o}}$ & $A$ & $A r$ & $H_{\mathrm{e}}$ & $H_{\mathrm{o}}$ & $A$ & $A r$ & $H_{\mathrm{e}}$ & $H_{\mathrm{o}}$ \\
\hline VKGP_202 & 2 & 1.948 & 0.091 & $0.000^{\text {**** }}$ & 2 & 1.839 & 0.071 & $0.000^{\text {**** }}$ & 2 & 2.000 & 0.391 & $0.000^{\text {**** }}$ & 3 & 2.357 & 0.184 & 0.000 \\
\hline VKGP_2694 & 4 & 3.454 & 0.279 & $0.136^{*}$ & 6 & 4.455 & 0.239 & $0.111^{\text {***** }}$ & 5 & 4.024 & 0.453 & 0.444 & 8 & 5.584 & 0.324 & 0.231 \\
\hline VKGP_3319 & 1 & 1.000 & 0.000 & 0.000 & 1 & 1.000 & 0.000 & 0.000 & 1 & 1.000 & 0.000 & 0.000 & 2 & 2.000 & 0.000 & 0.000 \\
\hline VKGP_4952 & 2 & 1.727 & 0.044 & 0.046 & 2 & 1.839 & 0.071 & 0.074 & 2 & 1.533 & 0.033 & 0.033 & 2 & 1.600 & 0.050 & 0.051 \\
\hline VKGP_10356 & 2 & 2.000 & 0.500 & $1.000^{\text {**** }}$ & 2 & 2.000 & 0.458 & $0.708^{* *}$ & 3 & 2.552 & 0.355 & 0.310 & 4 & 3.169 & 0.438 & 0.673 \\
\hline VKGP_14936 & 1 & 1.000 & 0.000 & 0.000 & 2 & 1.857 & 0.074 & $0.000^{\text {***** }}$ & 2 & 2.000 & 0.500 & $1.000^{* * * * *}$ & 3 & 2.373 & 0.191 & 0.333 \\
\hline VKGP_19219 & 2 & 1.948 & 0.091 & $0.000^{* * *}$ & 4 & 3.329 & 0.180 & 0.192 & 4 & 3.523 & 0.222 & $0.103^{* * * *}$ & 6 & 3.511 & 0.164 & 0.099 \\
\hline VKGP_25965 & 1 & 1.000 & 0.000 & 0.000 & 1 & 1.000 & 0.000 & 0.000 & 2 & 2.000 & 0.444 & $0.133^{\text {**** }}$ & 2 & 2.000 & 0.148 & 0.044 \\
\hline VKGP_26672 & 2 & 1.930 & 0.087 & $0.000^{\text {**** }}$ & 3 & 2.185 & 0.072 & 0.074 & 3 & 2.862 & 0.213 & 0.233 & 5 & 3.647 & 0.124 & 0.103 \\
\hline VKGP_29328 & 1 & 1.000 & 0.000 & 0.000 & 1 & 1.000 & 0.000 & 0.000 & 2 & 1.786 & 0.064 & 0.067 & 2 & 2.000 & 0.022 & 0.022 \\
\hline VKGP_31098 & 2 & 1.696 & 0.043 & 0.044 & 2 & 1.839 & 0.071 & 0.074 & 2 & 1.533 & 0.033 & 0.033 & 4 & 2.722 & 0.049 & 0.050 \\
\hline VKGP_31943 & 1 & 1.000 & 0.000 & 0.000 & 1 & 1.000 & 0.000 & 0.000 & 2 & 2.000 & 0.346 & $0.000^{\text {**** }}$ & 2 & 2.000 & 0.115 & 0.000 \\
\hline VKGP_32221 & 4 & 3.588 & 0.210 & $0.045^{\text {**** }}$ & 5 & 3.703 & 0.181 & $0.115^{* *}$ & 7 & 5.376 & 0.484 & 0.400 & 12 & 5.391 & 0.292 & 0.187 \\
\hline VKGP_87603 & 1 & 1.000 & 0.000 & 0.000 & 1 & 1.000 & 0.000 & 0.000 & 4 & 3.983 & 0.634 & $0.333^{*}$ & 5 & 4.743 & 0.211 & 0.111 \\
\hline VKGP_92431 & 3 & 2.608 & 0.124 & 0.130 & 3 & 2.531 & 0.139 & $0.074^{*}$ & 3 & 2.615 & 0.292 & 0.269 & 5 & 3.266 & 0.185 & 0.158 \\
\hline VKGP_108374 & 2 & 1.727 & 0.044 & 0.046 & 4 & 2.846 & 0.111 & 0.115 & 2 & 1.533 & 0.033 & 0.033 & 4 & 1.911 & 0.063 & 0.065 \\
\hline VKGP_108676 & 3 & 2.391 & 0.084 & 0.087 & 2 & 1.938 & 0.105 & 0.111 & 4 & 3.531 & 0.242 & 0.233 & 5 & 3.550 & 0.144 & 0.144 \\
\hline YGCX_17221 & 1 & 1.000 & 0.000 & 0.000 & 1 & 1.000 & 0.000 & 0.000 & 4 & 3.916 & 0.684 & $0.276^{* * *}$ & 6 & 5.461 & 0.228 & 0.092 \\
\hline YGCX_28878 & 4 & 3.390 & 0.268 & 0.217 & 4 & 2.778 & 0.107 & 0.111 & 6 & 4.318 & 0.215 & 0.231 & 9 & 3.498 & 0.197 & 0.186 \\
\hline Average & 2.1 & 1.864 & 0.098 & 0.092 & 2.5 & 2.060 & 0.099 & 0.093 & 3.2 & 2.741 & 0.297 & 0.217 & 4.7 & 3.199 & 0.165 & 0.134 \\
\hline
\end{tabular}

$A$, number of alleles per locus; $A r$, allelic richness; $H_{\mathrm{e}}$, expected heterozygosity; $H_{0}$, observed heterozygosity; $n$, number of individuals genotyped. Asterisks denote significant departure from Hardy-Weinberg equilibrium $\left({ }^{*} P<0.05,{ }^{* * *} P<0.01,{ }^{* * *} P<0.001\right)$. Petal color is indicated with population name. 
(Tokyo Pref.), 27 from Mifune (Kumamoto Pref.) and 30 from Nantan (Kyoto Pref.) was used to evaluate allelic polymorphisms. To characterize each microsatellite marker (= 19 markers), four summary statistics were calculated using FSTAT v.2.9.3 (Goudet, 1995) and GenAlEx v.6.501 (Peakall and Smouse, 2012): the number of alleles
$(A)$, allelic richness $(A r)$, expected heterozygosity $\left(H_{\mathrm{e}}\right)$ and observed heterozygosity $\left(H_{0}\right)$. These summary statistics were calculated for each locus and population. The significance of Hardy-Weinberg equilibrium and genotypic equilibrium were tested by chi-squared test using GenAlEx v.6.501. In addition, the $F_{\mathrm{ST}}$ index (Weir and Cockerham,

Table 3. A further 20 primer pairs that were monomorphic or fixed to a heterozygote genotype for G. thunbergii

\begin{tabular}{|c|c|c|c|c|c|c|}
\hline Locus & Primer sequence $\left(5^{\prime}-3^{\prime}\right)$ & $\begin{array}{l}\text { Predicted } \\
\text { size }(b p)\end{array}$ & Motif & BLASTX top hit description [species] & $E$-value & ONEKP ID \\
\hline VKGP_4891 & $\begin{array}{l}\text { F: CTCCAAGGTACGAGGTGGTC } \\
\text { R: GATGTCACGACGTTCACAGC }\end{array}$ & 345 & $(\mathrm{AAC})_{8}$ & $\begin{array}{l}\text { transcription repressor MYB4-like } \\
\text { [Rhodamnia argentea }]\end{array}$ & $6.00 \mathrm{E}-89$ & VKGP-2004891 \\
\hline VKGP_10465 & $\begin{array}{l}\text { F: CAGCATCGTTCTTTCCCACC } \\
\text { R: ACGTGCTGTTACAAACTGGG }\end{array}$ & 345 & $(\mathrm{AAG})_{13}$ & $\begin{array}{l}\text { LOW QUALITY PROTEIN: probable serine/threonine } \\
\text { protein kinase IRE4 [Pistacia vera] }\end{array}$ & $3.00 \mathrm{E}-68$ & VKGP-2010465 \\
\hline VKGP_12870 & $\begin{array}{l}\text { F: GGTTGGTTCTGTTTCTGGGC } \\
\text { R: CAACCAGCTCACACCTCAAC }\end{array}$ & 276 & $(\mathrm{AG})_{11}$ & $\begin{array}{c}\text { hypothetical protein CMV_011704 } \\
\text { [Castanea mollissima }]\end{array}$ & 0 & VKGP-2012870 \\
\hline VKGP_22151 & $\begin{array}{l}\text { F: TCATTGTGGCGAGCAAGTTC } \\
\text { R: TTGCCCGGGTTCTCTTATCC }\end{array}$ & 172 & $(\mathrm{AG})_{9}$ & No hits & & VKGP-2022151 \\
\hline VKGP_22086 & $\begin{array}{l}\text { F: CCAAATCACTGACCTCCACG } \\
\text { R: TCACAATCTCGTTCTCATCACC }\end{array}$ & 206 & $(\mathrm{AT})_{9}$ & $\begin{array}{l}\text { NAC domain-containing protein 43-like isoform X2 } \\
\text { [Hevea brasiliensis] }\end{array}$ & $3.00 \mathrm{E}-21$ & VKGP-2022086 \\
\hline VKGP_23492 & $\begin{array}{l}\text { F: CAACTTGATCATGCACTTGTGC } \\
\text { R: AGTCAGTGCTGGACAAGGAG }\end{array}$ & 289 & $(\mathrm{AAC})_{8}$ & $\begin{array}{c}\text { hypothetical protein G4B88_004180 } \\
\text { [Cannabis sativa }]\end{array}$ & $6.00 \mathrm{E}-129$ & VKGP-2023492 \\
\hline VKGP_23939 & $\begin{array}{l}\text { F: TTCGTCTGATTCGGCATTGC } \\
\text { R: TCGGCCATGGAAGGTAGAAG }\end{array}$ & 396,417 & $(\mathrm{AAG})_{8}$ & $\begin{array}{l}\text { mechanosensitive ion channel protein 10-like } \\
{[\text { Herrania umbratica }]}\end{array}$ & 0 & VKGP-2023939 \\
\hline VKGP_25427 & $\begin{array}{l}\text { F: AAATAGAGGGAACAAGGCGC } \\
\text { R: AGAGTATACGCCTCCATCGC }\end{array}$ & 188 & $(\mathrm{AG})_{9}$ & $\begin{array}{l}\text { AMP deaminase/myoadenylate deaminase, } \\
\text { putative isoform } 1 \text { [Theobroma cacao] }\end{array}$ & 0 & VKGP-2025427 \\
\hline VKGP_29076 & $\begin{array}{l}\text { F: ATCAGCCACCTCATCACCTC } \\
\text { R: TGGGCATGACGATATCCTGG }\end{array}$ & 230 & $(\mathrm{AAC})_{11}$ & No hits & & VKGP-2029076 \\
\hline VKGP_31603 & $\begin{array}{l}\text { F: GAGAACACAATCCTCGTCGC } \\
\text { R: AGCTCTCCTCCACTTCTTGC }\end{array}$ & 333 & $(\mathrm{AG})_{9}$ & hypothetical protein [Gossypium lobatum] & $2.00 \mathrm{E}-27$ & VKGP-2031603 \\
\hline VKGP_76520 & $\begin{array}{l}\text { F: TCTCACCGACCTTTCCCATC } \\
\text { R: CAGTCTCTAGTTGCTCATCAGG }\end{array}$ & 132 & $(\mathrm{AC})_{8}$ & No hits & & VKGP-2076520 \\
\hline VKGP_78116* & $\begin{array}{l}\text { F: GAGAGGCTTGCGATGGAGAG } \\
\text { R: AAAGCTCCACTCAACAACGC }\end{array}$ & 118 & $(\mathrm{AG})_{9}$ & No hits & & VKGP-2078116 \\
\hline YGCX_1175 & $\begin{array}{l}\text { F: GATTCTGCTTCTCGTGACCC } \\
\text { R: GAAGCTCACTGTCTCGTTGC }\end{array}$ & 176 & $(\mathrm{AAG})_{9}$ & $\begin{array}{c}\text { aldo-keto reductase family } 4 \text { member c9-like protein } \\
\text { [Trifolium pratense }]\end{array}$ & $5.00 \mathrm{E}-06$ & YGCX-2001175 \\
\hline YGCX_3345 & $\begin{array}{l}\text { F: TCCTCCTGTATCGCCGAAAG } \\
\text { R: CCCGAATCCATTTGAGGTGC }\end{array}$ & 225 & $(\mathrm{AGC})_{8}$ & unnamed protein product $[$ Prunus armeniaca $]$ & $2.00 \mathrm{E}-51$ & YGCX-2003345 \\
\hline YGCX_6368 & $\begin{array}{l}\text { F: CCCTTCCAACAAGTGCATGG } \\
\text { R: AGCTTCTGTGAGGGAGGAAC }\end{array}$ & 313 & $(\mathrm{AAC})_{8}$ & probable transcription factor PosF21 [Cicer arietinum] & $2.00 \mathrm{E}-08$ & YGCX-2006368 \\
\hline YGCX_22751 & $\begin{array}{l}\text { F: TCCTCTGAGCTATGGTGTCAC } \\
\text { R: ATCCCTCTCACAATCTGGCC }\end{array}$ & 270 & $(\mathrm{AAG})_{9}$ & $\begin{array}{l}\text { senescence/dehydration-associated protein At4g35985, } \\
\text { chloroplastic-like isoform X1 [Rosa chinensis] }\end{array}$ & $1.00 \mathrm{E}-114$ & YGCX-2022751 \\
\hline YGCX_22772 & $\begin{array}{l}\text { F: CTGATGAACTTGGACGACGC } \\
\text { R: ATGTGGAGAGGATCATGGCC }\end{array}$ & 392 & $(\mathrm{AAC})_{9}$ & $\begin{array}{l}\text { AP2-like ethylene-responsive transcription factor ANT } \\
\text { [Herrania umbratica }]\end{array}$ & $6.00 \mathrm{E}-36$ & YGCX-2022772 \\
\hline YGCX_23325 & $\begin{array}{l}\text { F: TTGAGCCGGAACAGAGTCAG } \\
\text { R: CGAGAATGTCACCGAACTGC }\end{array}$ & 265 & $(\mathrm{ACC})_{8}$ & RNA-binding protein 38 isoform X3 [Prosopis alba] & $2.00 \mathrm{E}-44$ & YGCX-2023325 \\
\hline YGCX_25909 & $\begin{array}{l}\text { F: GCCACTACAACTGGACTTGC } \\
\text { R: ATCTGCCCTATGAGCTCCAG }\end{array}$ & 404 & $(\mathrm{ATC})_{9}$ & No hits & & YGCX-2025909 \\
\hline YGCX_28044 & $\begin{array}{l}\text { F: ACCATCAATTTGCGGGACAC } \\
\text { R: GCACCAACATCATCCCTCTC }\end{array}$ & 208 & $(\mathrm{AAG})_{14}$ & $\begin{array}{l}\text { protein ENHANCED DISEASE RESISTANCE 2-like } \\
\text { [Carica papaya }]\end{array}$ & $4.00 \mathrm{E}-22$ & YGCX-2028044 \\
\hline
\end{tabular}

"This primer pair was previously reported as a monomorphic microsatellite primer pair of G. soboliferum var. kiusianum (Kurata et al., 2017). 
1984) between populations was calculated using FSTAT v.2.9.3 for elucidating genetic differentiation among the three populations. Cross-amplification trials of the 19 markers were also performed for the related species $G$. wilfordii Maxim. (16 individuals from two populations: Onneyu, Hokkaido Pref. and Oshino, Yamanashi Pref.) and G. sibiricum L. (16 individuals from two populations: Nakatonbetsu and Onneyu, Hokkaido Pref.).

For the first primer screening using the auto sequencer, 39 of 141 primer pairs successfully amplified DNA fragments of the predicted size, while the remaining 102 pairs amplified fragments of unpredicted size, produced multiple bands, or failed to amplify any fragment. For the 39 reliable primer pairs that showed clear microsatellite peaks of the predicted fragment size, we conducted a second PCR trial using 80 individuals from three populations. We found that 19 loci were polymorphic across the three populations (Table 1), ranging from two to 12 alleles with $H_{\mathrm{e}}$ and $H_{\mathrm{o}}$ values ranging from 0.0 to 0.684 and 0.0 to 1.000 , respectively (Table 2). Among these 19 loci, five markers (Table 1, Table 2) were found to be identical to sequences that were previously reported as monomorphic microsatellite primers of $G$. soboliferum var. kiusianum
(Kurata et al., 2017). Genetic diversity was highest in the Nantan population $\left(\mathrm{Ar}=2.741, H_{\mathrm{e}}=0.297\right)$, followed by the populations of Mifune $\left(A r=2.060, H_{\mathrm{e}}=0.099\right)$ and Hachioji ( $\mathrm{Ar}=1.864, H_{\mathrm{e}}=0.098$ ) (Table 2). In the Nantan population, individuals of both flower colors are distributed, while only purple and white flowers are distributed in Mifune and Hachioji, respectively. We confirmed significant departures from Hardy-Weinberg equilibrium at some loci in particular populations. Specifically, we detected significant deviations at VKGP_202, VKGP_2694, VKGP_10356, VKGP_14936, VKGP_19219 and VKGP_32221 in two or three populations in each locus, which may indicate the presence of null alleles at these loci. Significant genetic differentiation among the three $G$. thunbergii populations was detected using these markers (i.e., Hachioji-Mifune, $F_{\mathrm{ST}}=0.841$; HachiojiNantan, $F_{\mathrm{ST}}=0.574$; Mifune-Nantan, $F_{\mathrm{ST}}=0.647$ ). The genotyping error rate of the 19 markers was $2.18 \%$ based on 24 individuals arbitrarily selected from three populations. Note that the remaining 20 primers were inappropriate for performing population genetic studies because all individuals were fixed to an allele (i.e., monomorphic) or a heterozygote genotype (Table 3 ).

Table 4. Cross-amplification results of the 19 markers for G. wilfordii and G. sibiricum

\begin{tabular}{|c|c|c|c|c|c|c|c|c|c|c|c|c|c|c|c|c|}
\hline \multirow{3}{*}{ Locus } & \multicolumn{8}{|c|}{ G. wilfordii } & \multicolumn{8}{|c|}{ G. sibiricum } \\
\hline & \multicolumn{4}{|c|}{ Onneyu $(n=8)$} & \multicolumn{4}{|c|}{ Oshino $(n=8)$} & \multicolumn{4}{|c|}{ Nakatonbetsu $(n=8)$} & \multicolumn{4}{|c|}{ Onneyu $(n=8)$} \\
\hline & $A$ & $A r$ & $H_{\mathrm{e}}$ & $H_{\mathrm{o}}$ & $A$ & $A r$ & $H_{\mathrm{e}}$ & $H_{\mathrm{o}}$ & $A$ & $A r$ & $H_{\mathrm{e}}$ & $H_{\mathrm{o}}$ & $A$ & $A r$ & $H_{\mathrm{e}}$ & $H_{\mathrm{o}}$ \\
\hline VKGP_202 & 2 & 1.992 & 0.219 & 0.000 & 1 & 1.000 & 0.000 & 0.000 & 1 & 1.000 & 0.000 & 0.000 & 1 & 1.000 & 0.000 & 0.000 \\
\hline VKGP_2694 & 3 & 2.867 & 0.320 & 0.250 & 3 & 2.875 & 0.398 & 0.125 & 2 & 1.875 & 0.117 & 0.125 & 1 & 1.000 & 0.000 & 0.000 \\
\hline VKGP_3319 & 3 & 3.000 & 0.586 & 0.125 & 2 & 2.000 & 0.375 & 0.250 & 1 & 1.000 & 0.000 & 0.000 & 2 & 2.000 & 0.245 & 0.000 \\
\hline VKGP_4952 & 2 & 1.875 & 0.117 & 0.125 & 3 & 2.750 & 0.227 & 0.250 & 2 & 1.875 & 0.117 & 0.125 & 1 & 1.000 & 0.000 & 0.000 \\
\hline VKGP_10356 & 2 & 1.875 & 0.117 & 0.125 & 3 & 2.750 & 0.227 & 0.250 & 1 & 1.000 & 0.000 & 0.000 & 3 & 2.875 & 0.398 & 0.250 \\
\hline VKGP_14936 & 2 & 1.875 & 0.117 & 0.125 & 1 & 1.000 & 0.000 & 0.000 & 2 & 2.000 & 0.500 & 1.000 & 2 & 2.000 & 0.500 & 1.000 \\
\hline VKGP_19219 & 3 & 3.000 & 0.255 & 0.286 & 2 & 1.875 & 0.117 & 0.125 & 2 & 2.000 & 0.245 & 0.000 & 2 & 2.000 & 0.133 & 0.143 \\
\hline VKGP_25965 & 1 & 1.000 & 0.000 & 0.000 & 1 & 1.000 & 0.000 & 0.000 & 1 & 1.000 & 0.000 & 0.000 & - & - & - & - \\
\hline VKGP_26672 & 2 & 1.992 & 0.219 & 0.000 & 2 & 1.875 & 0.117 & 0.125 & 1 & 1.000 & 0.000 & 0.000 & 2 & 1.875 & 0.117 & 0.125 \\
\hline VKGP_29328 & 2 & 1.875 & 0.117 & 0.125 & 3 & 3.000 & 0.633 & 0.250 & 1 & 1.000 & 0.000 & 0.000 & 1 & 1.000 & 0.000 & 0.000 \\
\hline VKGP_31098 & 3 & 2.867 & 0.320 & 0.375 & 4 & 3.625 & 0.328 & 0.375 & 3 & 2.750 & 0.227 & 0.250 & 3 & 2.750 & 0.227 & 0.250 \\
\hline VKGP_31943 & 2 & 2.000 & 0.430 & 0.125 & 2 & 2.000 & 0.305 & 0.125 & 1 & 1.000 & 0.000 & 0.000 & 1 & 1.000 & 0.000 & 0.000 \\
\hline VKGP_32221 & 2 & 2.000 & 0.500 & 1.000 & 2 & 2.000 & 0.500 & 1.000 & 1 & 1.000 & 0.000 & 0.000 & 2 & 1.875 & 0.117 & 0.125 \\
\hline VKGP_87603 & 2 & 2.000 & 0.305 & 0.125 & 2 & 2.000 & 0.492 & 0.125 & 1 & 1.000 & 0.000 & 0.000 & 1 & 1.000 & 0.000 & 0.000 \\
\hline VKGP_92431 & 3 & 2.867 & 0.320 & 0.125 & 1 & 1.000 & 0.000 & 0.000 & 2 & 1.992 & 0.219 & 0.000 & 1 & 1.000 & 0.000 & 0.000 \\
\hline VKGP_108374 & 2 & 1.992 & 0.219 & 0.250 & 2 & 1.875 & 0.117 & 0.125 & 2 & 1.875 & 0.117 & 0.125 & 2 & 1.875 & 0.117 & 0.125 \\
\hline VKGP_108676 & 1 & 1.000 & 0.000 & 0.000 & 4 & 3.858 & 0.492 & 0.375 & 2 & 1.992 & 0.219 & 0.000 & 3 & 2.867 & 0.320 & 0.375 \\
\hline YGCX_17221 & 2 & 1.875 & 0.117 & 0.125 & 1 & 1.000 & 0.000 & 0.000 & 1 & 1.000 & 0.000 & 0.000 & 1 & 1.000 & 0.000 & 0.000 \\
\hline YGCX_28878 & 2 & 1.992 & 0.219 & 0.000 & 2 & 1.875 & 0.117 & 0.125 & 2 & 1.875 & 0.117 & 0.125 & 2 & 1.875 & 0.117 & 0.125 \\
\hline Average & 2.2 & 2.102 & 0.237 & 0.173 & 2.2 & 2.071 & 0.234 & 0.191 & 1.5 & 1.486 & 0.099 & 0.092 & 1.7 & 1.666 & 0.127 & 0.140 \\
\hline
\end{tabular}

$A$, number of alleles per locus; $A r$, allelic richness; $H_{\mathrm{e}}$, expected heterozygosity; $H_{\mathrm{o}}$, observed heterozygosity; $n$, number of individuals genotyped. One marker, VKGP_25965, failed to PCR-amplify any fragments for G. sibiricum. 
Moreover, we checked for cross-amplification of these polymorphic markers in G. wilfordii and G. sibiricum. In G. wilfordii, although one locus (VKGP_25965) was monomorphic, the other 18 loci were polymorphic across the two populations, ranging from two to four alleles with $H_{\mathrm{e}}$ and $H_{\mathrm{o}}$ values ranging from 0.0 to 0.633 and 0.0 to 1.000, respectively (Table 4). Allelic richness $(A r)$ ranged from 1.000 to 3.858 (Table 4). In G. sibiricum, 13 markers showed polymorphisms across the two populations, with the number of alleles ranging from two to three and $H_{\mathrm{e}}$ and $H_{\mathrm{o}}$ values ranging from 0.0 to 0.500 and 0.0 to 1.000 , respectively (Table 4). Allelic richness (Ar) ranged from 1.000 to 2.875 (Table 4). However, six loci (i.e., VKGP_202, VKGP_25965, VKGP_29328, VKGP_31943, VKGP_87603 and YGCX_17221) were monomorphic, and one marker, VKGP_25965, failed to PCR-amplify any fragments for $G$. sibiricum.

Overall, the microsatellite markers developed here will be useful to reveal the genetic structure and demographic history of $G$. thunbergii in the Japanese archipelago, which will elucidate the genetic background of flower color polymorphism among populations.

The authors thank Daiki Takahashi, Kazutoshi Masuda and Koki Nagasawa for their great help with the sampling. The authors are grateful to Drs. Atsushi Ohwaki and Kenji Horie for granting access to their collection of materials (G. wilfordii and G. sibiricum). We are grateful to the Ashiu Forest Research Station (Kyoto University) for granting us permission to perform field surveys. We are also grateful to the ONEKP: BLAST for 1,000 Plants for kindly allowing us to access the sequence data. This work was supported by JSPS KAKENHI under Grant No. JP16H04827; the Agency for Medical Research and Development National BioResource Project under Grant No. 18km0210136j0002; and Fujiwara Natural History Foundation.

\section{REFERENCES}

Akiyama, S. (2001) Geraniaceae. In Flora of Japan, Vol. IIb. (eds.: Iwatsuki, K., Boufford, D. E., and Ohba, H.), p. 289. Kodansha Ltd., Tokyo, Japan.

Altschul, S. F., Gish, W., Miller, W., Myers, E. W., and Lipman, D. J. (1990) Basic local alignment search tool. J. Mol. Biol. 215, 403-410.

Arista, M., Talavera, M., Berjano, R., and Ortiz, P. L. (2013) Abiotic factors may explain the geographical distribution of flower colour morphs and the maintenance of colour polymorphism in the scarlet pimpernel. J. Ecol. 101, 16131622

Brownstein, M. J., Carpten, J. D., and Smith, J. R. (1996) Modulation of non-templated nucleotide addition by Taq DNA polymerase: primer modifications that facilitate genotyping. Biotechniques 20, 1004-1010.
Darwin, C. (1862) On the Various Contrivances by Which British and Foreign Orchids are Fertilised by Insects, and on the Good Effects of Intercrossing. John Murray, London, UK.

Faircloth, B. C. (2008) MSATCOMMANDER: detection of microsatellite repeat arrays and automated, locus-specific primer design. Mol. Ecol. Resour. 8, 92-94.

Forsman, A. (2016) Is colour polymorphism advantageous to populations and species? Mol. Ecol. 25, 2693-2698.

Gigord, L. D. B., Macnair, M. R., and Smithson, A. (2001) Negative frequency-dependent selection maintains a dramatic flower color polymorphism in the rewardless orchid Dactylorhiza sambucina (L.) Soó. Proc. Natl. Acad. Sci. USA 98, 6253-6255.

Goudet, J. (1995) FSTAT (Version 1.2): A computer program to calculate $F$-statistics. J. Hered. 86, 485-486.

Jones, K. N., and Reithel, J. S. (2001) Pollinator-mediated selection on a flower color polymorphism in experimental populations of Antirrhinum (Scrophulariaceae). Am. J. Bot. 88, 447-454.

Kadota, Y. (2016) Geraniaceae. In Wild flowers of Japan, Vol. 3. (eds.: Ohashi, H., Kadota, Y., Murata, J., Yonekura, K., and Kihara, H.), pp. 252-253. Heibonsha, Tokyo, Japan (in Japanese).

Kurata, S., Sakaguchi, S., and Ito, M. (2017) Development of nuclear microsatellite markers for the threatened wetland plant Geranium soboliferum var. kiusianum (Geraniaceae). Plant Species Biol. 32, 466-470.

McCall, A. C., Murphy, S. J., Venner, C., and Brown, M. (2013) Florivores prefer white versus pink petal color morphs in wild radish, Raphanus sativus. Oecologia 172, 189-195.

Milligan, B. (1992) Plant DNA isolation. In Molecular Genetic Analysis of Populations: A Practical Approach, 2nd ed. (ed.: Hoelzel, A. R.), pp. 59-88. IRL Press, Oxford, UK.

Narbona, E., Wang, H., Ortiz, P. L., Arista, M., and Imbert, E. (2018) Flower colour polymorphism in the Mediterranean Basin: occurrence, maintenance and implications for speciation. Plant Biol. 20, 8-20.

Peakall, R., and Smouse, P. E. (2012) GenAlEx 6.5: genetic analysis in Excel. Population genetic software for teaching and research-an update. Bioinformatics 28, 2537-2539.

Rozen, S., and Skaletsky, H. (2000) Primer3 on the WWW for general users and for biologist programmers. Methods Mol. Biol. 132, 365-386.

Thairu, M. W., and Brunet, J. (2015) The role of pollinators in maintaining variation in flower colour in the Rocky Mountain columbine, Aquilegia coerulea. Ann. Bot. 115, 971979.

Tsuchimatsu, T., Yoshitake, H., and Ito, M. (2014) Herbivore pressure by weevils associated with flower color polymorphism in Geranium thunbergii (Geraniaceae). J. Plant Res. 127, 265-273.

Weir, B. S., and Cockerham, C. C. (1984) Estimating $F$-statistics for the analysis of population structure. Evolution 38, $1358-1370$

Wright, S. (1978) Evolution and the Genetics of Populations, Vol. 4. Variability Within and Among Natural Populations. University of Chicago Press, Chicago, USA. 